\title{
Sind 82 Rappen Taxpunktwert noch vertretbar?
}

\section{Daniel Jud}

Für den Vorstand der Ärztegesellschaft Thurgau
An der Mitgliederversammlung der Ärztegesellschaft Thurgau wurde diskutiert, ob der regionale Anschlussvertrag zum Rahmenvertrag TARMED gekündigt werden soll. Da die Tarifregion Ostschweiz die Entscheide in den Kantonen Aargau und Graubünden abwarten will, bevor Massnahmen ergriffen werden, war für die Ärztegesellschaft Thurgau nur ein Alleingang denkbar.

Der allgemeine Tenor an der Versammlung war ganz klar eine grosse Unzufriedenheit mit dem seit 2007 stagnierten Taxpunktwert von 82 Rappen. Die mit konkreten Daten aus den Arztpraxen erstellten Berechnungen von NewIndex zeigen eindeutig auf, dass die Kosten seit 2007 beachtlich gestiegen sind.
Insgesamt haben wir seit 1992 einen eingefrorenen Tarif, der dann kostenneutral in den TARMED überführt wurde. Alle Schweizerinnen und Schweizer haben Anrecht auf Teuerungsausgleich, der uns Ärzten nicht gewährt wird. Somit werden die Gestehungskosten in der ärztlichen Versorgung (insbesondere auch der Grundversorgung) immer grösser und der aktuelle Taxpunktwert entbehrt jeglicher betriebswirtschaftlichen Logik, die für alle produzierenden Betriebe in unserem Lande als Notwendigkeit zugrunde gelegt wird (vgl. 1.20 CHF als Untergrenze beim Euro).

Ganz konkret leiden gewisse Regionen im Kanton Thurgau unter einer so massiven Unterversor-

\section{Alle Schweizerinnen und Schweizer haben Anrecht auf Teuerungsausgleich.}

Korrespondenz:

Dr. med. Daniel Jud

Präsident der Ärztegesellschaft Thurgau

Weinfelderstrasse 4

CH-8580 Amriswil

daniel.jud[at]hin.ch
Gemäss der Modellrechnung würde der Taxpunktwert für die Ostschweiz unter Berücksichtigung der Teuerung und der höheren Löhne für Angestellte bei 93 Rappen liegen. Die anwesenden Ärztinnen und Ärzte beauftragten den Vorstand, sämtliche Möglichkeiten zur Erhöhung des Taxpunktwertes zu prüfen und den allgemeinen Unmut kundzutun.

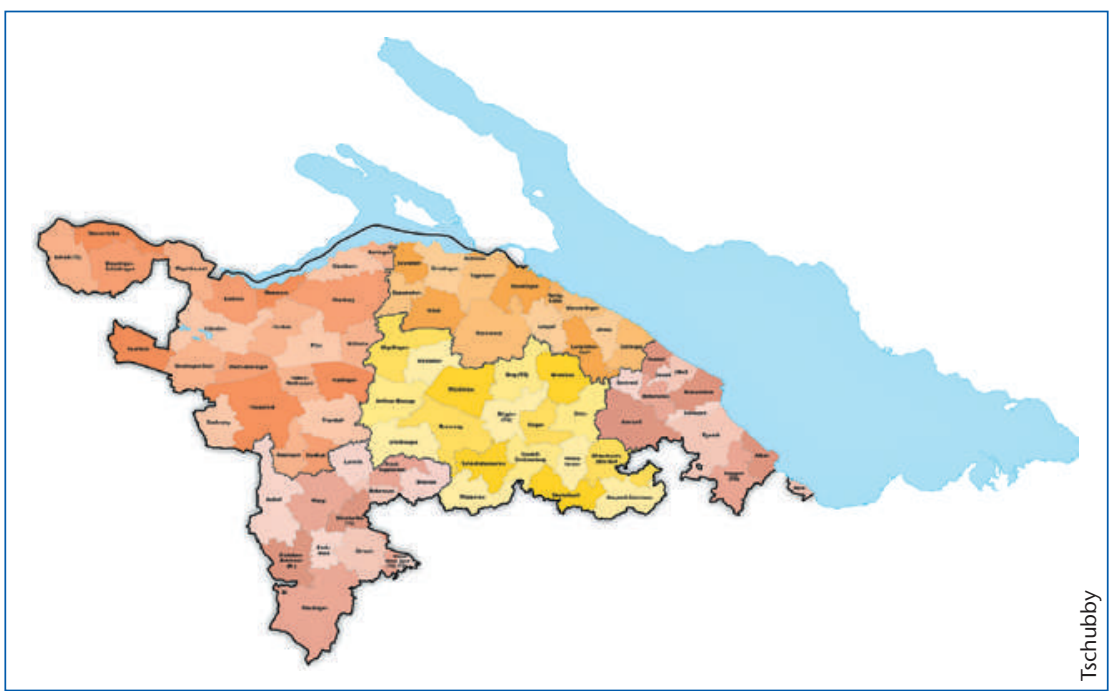

Einige Regionen im Kanton Thurgau leiden unter so massiver Unterversorgung bei den Grundversorgern, dass die Bevölkerung kaum mehr angemessen behandelt werden kann.

gung von Grundversorgern, dass die Bevölkerung kaum mehr angemessen behandelt werden kann. Neue Hausärzte haben einen sehr schweren Stand, sind sie doch in der Regel gar nicht mehr in der Lage, die notwendigen Investitionen zu tätigen. Die Versorgungsabklärung der Ärztegesellschaft Thurgau hat zudem eine Unterversorgung bei den Psychiaterinnen und Psychiatern gezeigt. Diese Berufsgruppe mit den tiefsten Ärzteeinkommen hat häufig Mühe, eine angemessene Altersvorsorge aufzubauen. Die an der Realität vorbeizielenden Vorstellungen der Krankenkassen und des Preisüberwachers stossen auf grosses Unverständnis, sollte eine gute ärztliche Versorgung doch für alle zentral sein. Unverständlich ist insbesondere, weshalb derselbe Preis für eine Leistung gelten soll, die in der Zwischenzeit verbessert wurde und die Leistungserbringer mehr kostet.

Da die Kündigung des Regionalen Anschlussvertrages im Alleingang für die Ärztegesellschaft Thurgau den Wegfall der regionalen und nationalen LeiKoV und damit eine zentrale Rolle der unberechenbaren Vorschläge des Preisüberwachers bedeutet hätte, sieht die Ärztegesellschaft Thurgau dieses Jahr noch von der Kündigung der Verträge ab. Aufgrund des klaren Auftrages der Mitglieder werden aber ein Vorgehen im grösseren Verbund sowie ein Alleingang per Ende Dezember 2013 geprüft und angestrebt. 